\title{
Plasmon-enhanced high-harmonic generation from silicon
}

\author{
G. Vampa ${ }^{1,2 \star}$, B. G. Ghamsari ${ }^{1,3}$, S. Siadat Mousavi ${ }^{1}$, T. J. Hammond ${ }^{1}$, A. Olivieri ${ }^{1}$, E. Lisicka-Skrek ${ }^{1}$, \\ A. Yu Naumov ${ }^{3}$, D. M. Villeneuve ${ }^{3}$, A. Staudte ${ }^{3}$, P. Berini ${ }^{1}$ and P. B. Corkum ${ }^{1,3 \star}$
}

\begin{abstract}
Plasmonic antennas can enhance the intensity of a nanojoule laser pulse by localizing the electric field in their proximity'. It has been proposed that the field can become strong enough to convert the fundamental laser frequency into high-order harmonics through an extremely nonlinear interaction with gas atoms that occupy the nanoscopic volume surrounding the antennas ${ }^{2-4}$. However, the small number of gas atoms that can occupy this volume limits the generation of high harmonics $^{5-7}$. Here we use an array of monopole nano-antennas to demonstrate plasmon-assisted high-harmonic generation directly from the supporting crystalline silicon substrate. The high density of the substrate compared with a gas allows macroscopic buildup of harmonic emission. Despite the sparse coverage of antennas on the surface, harmonic emission is ten times brighter than without antennas. Imaging the high-harmonic radiation will allow nanometre and attosecond measurement of the plasmonic field ${ }^{8}$ thereby enabling more sensitive plasmon sensors ${ }^{9}$ while opening a new path to extreme-ultraviolet-frequency combs ${ }^{10}$.
\end{abstract}

Traditional high-harmonic generation occurs in rare-gas atoms $^{11,12}$. Therefore, early experiments with plasmonic antennas ${ }^{2}$ or funnels ${ }^{3}$ assumed that high harmonics were generated through local field enhancement in the gas surrounding the nanostructures. However, considerable enhancement is also achieved below and around the antennas, in the material that supports them, as demonstrated in Supplementary Fig. 1.

In recent years, high harmonics have also been generated without nanostructures directly from the bulk of a $\mathrm{ZnO}$ crystal $^{13}$, and subsequently from various other materials ${ }^{14-16}$-in some cases with peak intensities as low as $10^{11}-10^{12} \mathrm{~W} \mathrm{~cm}^{-2}$. These intensities are two to three orders of magnitude lower than those required in the gas phase. Not only is the threshold intensity to drive this nonlinear optical process lower than in gases, but the density of solids is $\sim 1,000$ times greater. Hence, generating plasmon-assisted high harmonics within a crystalline substrate should overcome the limit of previous experiments (that is, the need to increase the number of emitters), and improve the longevity of the nanostructures to highpower irradiation (the longevity depends on their structural quality). These advantages have been exploited in a recent experiment conducted on Au-coated sapphire nano-cones ${ }^{17}$, where the surface plasmon is adiabatically excited-rather than resonantly excited as in this letter.

Figure 1a shows a sketch of the experimental setup (see also Supplementary Information). We focus infrared femtosecond laser pulses with a central wavelength of $2.1 \mu \mathrm{m}$ onto an array of $\mathrm{Au}$ monopolar nano-antennas fabricated on a thin film of singlecrystal $\mathrm{Si}$ (500 nm thick) grown on an $\mathrm{Al}_{2} \mathrm{O}_{3}$ single-crystal substrate
( $0.5 \mathrm{~mm}$ thick). Figure $1 \mathrm{~b}$ shows a high-resolution scanning electron micrograph of the structures (design and fabrication details are reported in the Supplementary Information). Two designs are implemented, with the antennas parallel to either the [110] or the [100] orientation of the Si crystal (therefore, rotated by $45^{\circ}$ ).

Figure 2a shows the high-harmonic spectrum produced by antennas in the configuration parallel to the [110] Si lattice. The emission extends from the 5 th to the 9 th harmonic for an incident intensity (in vacuum) of $3 \times 10^{10} \mathrm{~W} \mathrm{~cm}^{-2}$. The 11 th harmonic, at $190 \mathrm{~nm}$, lies beyond the detectable spectral range of our experimental setup. When the laser polarization is parallel to the antennas (red line), the high-harmonic emission is 5 to 10 times stronger than for polarization orthogonal to the antennas (green line), a configuration that does not lead to field enhancement (see Supplementary Fig. 1). In fact, in the latter case, the high-harmonic power spectrum is almost identical to that emitted from an area without any nano-antennas (black line). Parallel and perpendicular polarizations correspond to equivalent crystal directions; therefore, harmonic emission from bulk silicon is unchanged. Despite the small area covered by the antennas (8\%), and the small volume over which the field is enhanced, emission from the array is stronger than the emission from the bulk, that is, off the array (black line). We estimate that the high-harmonic emission is confined to regions of $\sim 20 \times 20 \mathrm{~nm}^{2}$ on either side of each antenna, and that the high-harmonic emission density in these regions is increased by $\sim 3 \times 10^{3}-3 \times 10^{4}$ times with respect to the bulk (see Supplementary Information).

In Fig. $2 b$ we show the intensity dependence of the harmonic radiation yield. Emission from antennas whose axis is parallel to the [100] direction (coloured diamonds in Fig. 2b) is weaker than for those aligned parallel to the [110] direction (coloured circles in Fig. 2b), as similarly measured for the bulk ${ }^{16}$ (compare grey circles with grey diamonds in Fig. 2b). Harmonic emission from Au would be irrespective of the orientation of the substrate. Further proof that the emission occurs from $\mathrm{Si}$ rather than $\mathrm{Au}$ is reported in Supplementary Fig. 3, which shows an image of the 5th harmonic generated from $\mathrm{Si}$ (bright areas) and not from micrometre-sized thin Au electrodes (black areas).

For antennas parallel to the [110] direction, all harmonics from the bulk scale approximately as $I^{3.5}$, where $I$ is the incident laser intensity, whereas perturbative nonlinear optics predicts $I^{n}$, where $n$ is the harmonic order ${ }^{18}$. A slightly slower scaling, also nonperturbative, has been measured from bulk $\mathrm{ZnO}$ (ref. 13). All harmonic orders emitted from the array (coloured lines) show the same intensity dependence, scaling approximately with $I^{3}$. This suggests that they are also generated non-perturbatively. The different scaling can arise from saturation of ionization above a threshold intensity (as found for $\mathrm{ZnO}$; ref. 13): because of enhancement, emission from

\footnotetext{
${ }^{1}$ University of Ottawa, Ottawa, Ontario K1N 6N5, Canada. ${ }^{2}$ PULSE Institute, SLAC National Accelerator Laboratory, Menlo Park, California 94025, USA.

${ }^{3}$ National Research Council of Canada, Ottawa, Ontario K1A OR6, Canada. ^e-mail: gvampa@stanford.edu; pcorkum@uottawa.ca
} 


\section{a $\quad 2,100 \mathrm{~nm}$} $100 \mathrm{fs}$
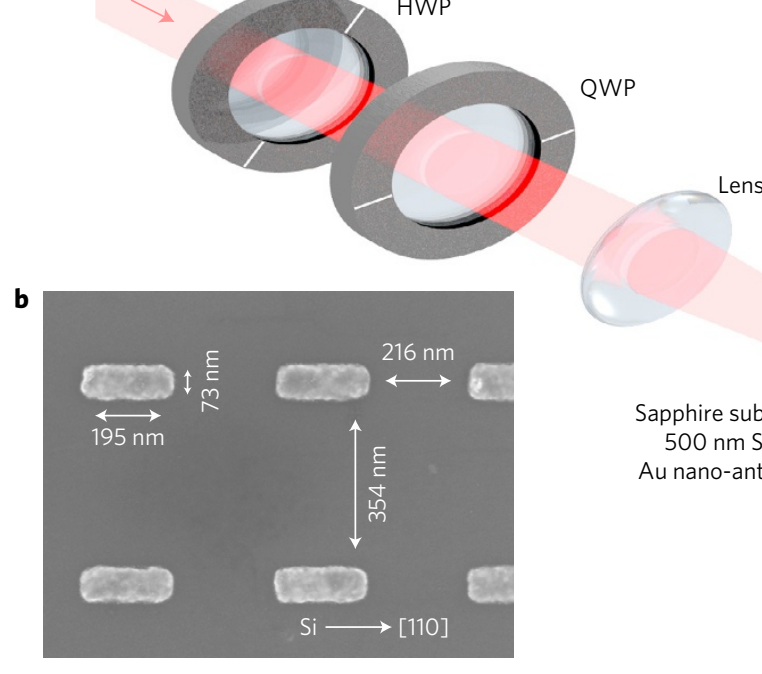
ens

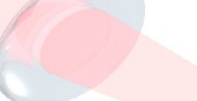

(1)

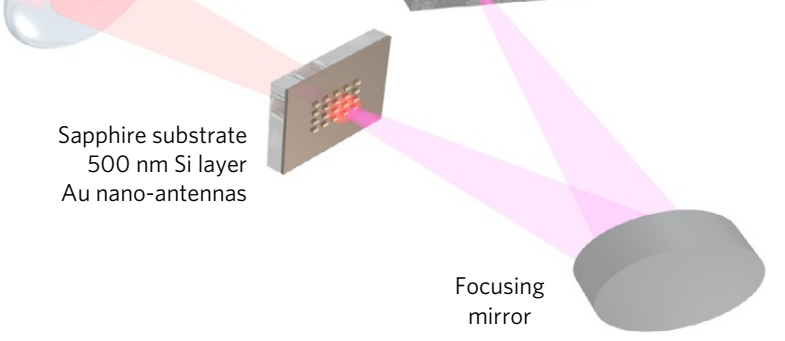

Figure 1 | Experimental setup. a, Laser pulses with a duration of $100 \mathrm{fs}$ and with a centre wavelength of $2.1 \mu \mathrm{m}$ are focused with a CaF 2 lens on an array of Au monopolar nano-antennas. The antennas are fabricated on a 500-nm-thick single-crystal Si film grown over a 500 um sapphire substrate (M-plane cut). A combination of a half-wave plate (HWP) and a quarter-wave plate (QWP) compensates for the birefringence of the sapphire substrate to achieve horizontal linear polarization inside the Si film. b. High-resolution scanning electron micrograph of an Au nano-antenna array. The antenna major axis is aligned along the [110] direction of the Si crystal, which yields the strongest high-harmonic emission from bulk Si. The length, width and height of the

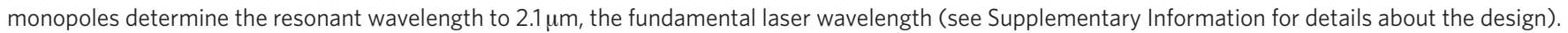

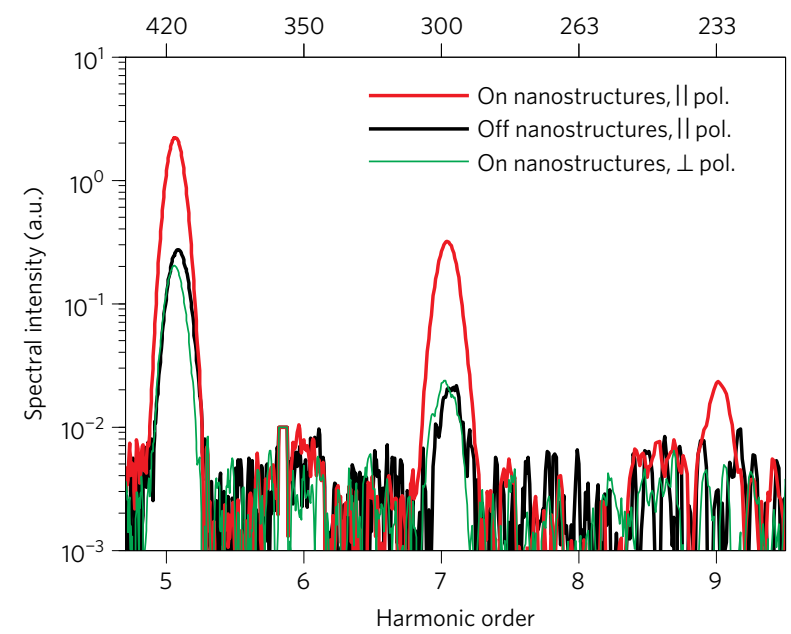

b

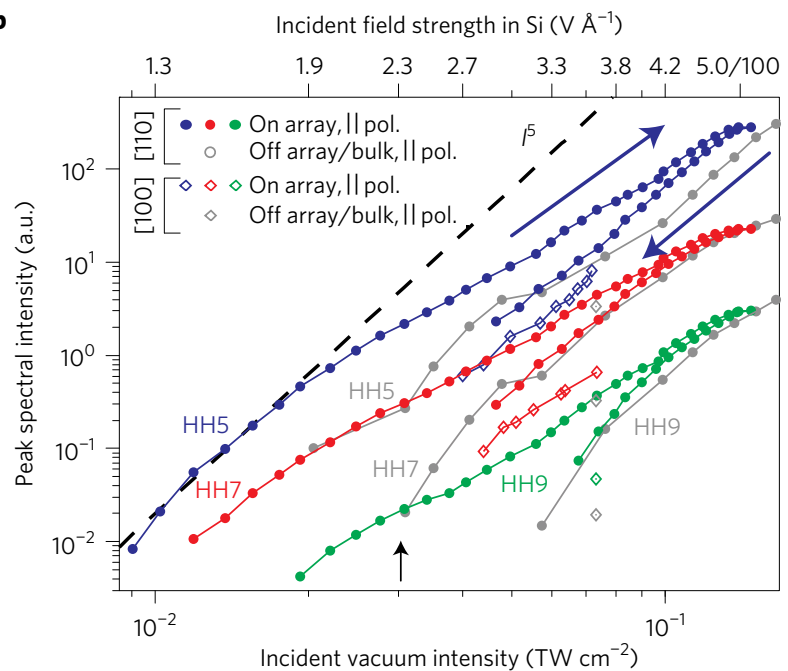

Figure 2 | Non-perturbative high-harmonic spectrum. a, High-harmonic spectrum extending up to the 9th harmonic ( $233 \mathrm{~nm}$ ), when antennas aligned along the [110] direction are illuminated with a polarization parallel (red line) and perpendicular (green line) to the major axis of the antennas. The black line is the emission from bulk $\mathrm{Si}$, when the sample is illuminated on the unpatterned material beside the array. The detection limit of our spectrometer extends to $200 \mathrm{~nm}$, which is not enough to measure the 11th harmonic (at $190 \mathrm{~nm}$ ). The vacuum intensity of the infrared driver is $3 \times 10^{10} \mathrm{~W} \mathrm{~cm}^{-2}$. $\mathbf{b}$, The peak harmonic signal from an array of antennas with their axis parallel to the [110] direction of the Si crystal (coloured circles) and from the bulk (grey circles), for polarization parallel to the axis of the antennas, is plotted as a function of the intensity of the infrared laser. The On/Off contrast decreases with increasing laser intensity (blue arrow pointing up-right), and remains close to unity for decreasing laser intensity (blue arrow pointing down-left). The scaling of bulk harmonics is reversible (not shown). When the axis of the antennas is parallel to the [100] direction instead, harmonic emission is weaker (open diamonds with coloured edges). Bulk emission from the [100] direction (open diamonds with grey edges) is also similarly weaker than from [110], suggesting that harmonics are emitted from Si rather than from Au. All harmonics scale non-perturbatively. The dashed black line, which scales with $I^{5}$, is a guide to the eye.

the array corresponds to a higher intensity inside $\mathrm{Si}$, which results in slower scaling. Alternatively, it may also arise from progressive damage to the antennas, as explained below.

The contrast between 'on' and 'off the array' steadily decreases with increasing laser intensity, approaching unity at the highest intensity, and then remains close to one for decreasing laser intensity. We interpret this irreversible behaviour as being due to progressive damage, but it is unclear whether it occurs to the Si or to the antennas (the antennas do not seem to be obviously altered, see Supplementary Fig. 6). Harmonics from unpatterned Si behave reversibly. In higher-bandgap materials, the intensity required for high-harmonic generation increases, and extensive damage to the antennas is expected ${ }^{19,20}$. However, generating harmonics from the substrate, rather than from gas atoms placed near the antennas, 
a

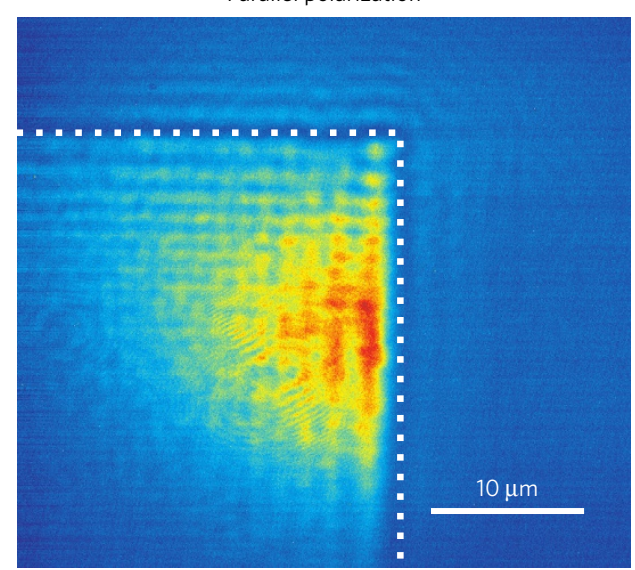

b

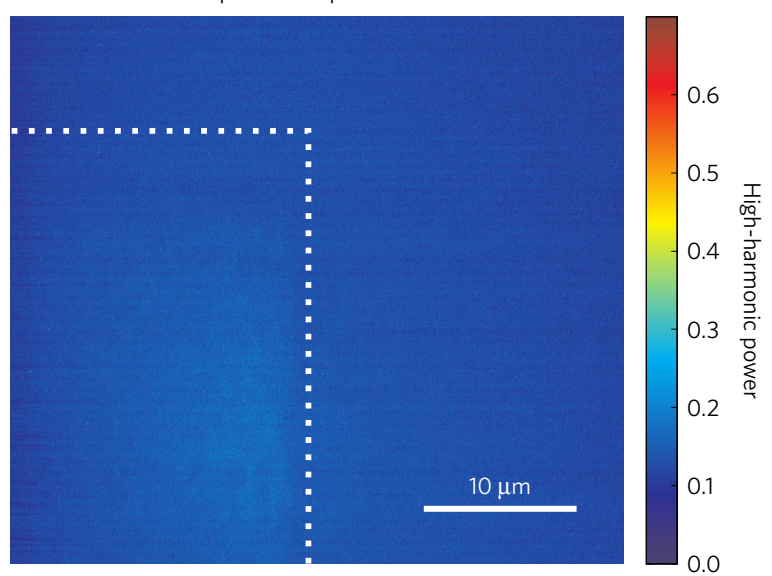

Figure $\mathbf{3}$ | Imaging high-harmonic emission. Image with the 5th harmonic of a corner of an illuminated array. a, The harmonic is emitted only for the portion of the beam that overlaps with the array and for polarization parallel to the antennas' major axis-a proof of field enhancement. $\mathbf{b}$, The emission

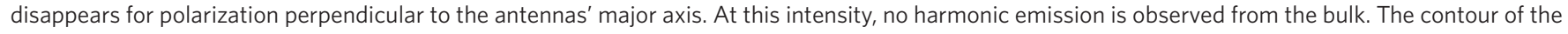
array is marked by the white dotted line.

offers opportunities to overcome the damage limit by encapsulating the antennas in a transparent dielectric medium, such as $\mathrm{SiO}_{2}$ or $\mathrm{MgF}_{2}$. Alternatively, the antennas can be buried in the Si film or fabricated with materials more resilient than $\mathrm{Au}$, such as TiN (ref. 21).

The diffraction of the high-harmonic beam carries information about the spatial distribution of nanoscopic emission regions. The simulations predict an inhomogeneous field enhancement, which is strong at the ends of the antennas and all along the edges (see Supplementary Fig. 1). Although these tiny features cannot be imaged with the infrared beam (unless electrons ${ }^{22}$ or near-field probes ${ }^{23}$ are used), they should be distinguishable with a short-wavelength highharmonic beam, whose diffraction limit of $\lambda_{\mathrm{HH}} / 2$ approaches $100 \mathrm{~nm}$ ( $\lambda_{\mathrm{HH}}$ is the wavelength of a high harmonic order). Figure 3 shows a corner of the array imaged with a magnification of 160 taken using a lens with $\mathrm{NA}=0.75$ and the 5 th harmonic $(\lambda=420 \mathrm{~nm})$, for polarization of the driving field parallel (Fig. 3a) and perpendicular (Fig. 3b) to the axis of the antennas. The emission is clearly brighter over the array for parallel polarization-another indication of enhancement. The blurring of the sharp edge of the array can be used to estimate a resolution of $\sim 1.6 \mu \mathrm{m}$ (4.6 times larger than the expected diffraction limit, defined by the Rayleigh criterion $r=0.61 \lambda / \mathrm{NA}$ ). This resolution is not sufficient to resolve individual nano-antennas. A larger-numerical-aperture lens should allow individual regions of field enhancement to be imaged. Lens-less imaging can also be used to exceed the diffraction limit ${ }^{24,25}$.

Our results have a number of important implications. First, because high-harmonic emission lasts only a fraction of the optical cycle, imaging the high harmonics can map the sub-cycle dynamics of the collective electronic motion, which influence the temporal ${ }^{8}$ and spatial properties ${ }^{26}$ of the local plasmonic field. For example, if the antennas are resonant with a 'probing field' that excites plasma oscillations, then the resulting local field will perturb the highharmonic generation process driven by a non-resonant driver. The perturbation will alter the spatial ${ }^{27}$ or spectral ${ }^{28,29}$ properties of the harmonic beam. By measuring the beam, we will be able to track the spatial evolution of the local field with attosecond temporal resolution.

Second, the interference between near field and bulk harmonics will contain a wealth of information about the fields in the antennas. For example, as the laser wavelength is scanned across the plasmonic resonance, the amplitude and phase of the near field will be modified relative to the bulk-which is largely unaffected by the frequency sweep. Each harmonic created in the array will inherit the phase shift of the plasmonic field, multiplied by the harmonic order. Thus, this homodyne detection scheme offers a highly sensitive method for studying plasmon responses that differs from previously proposed methods ${ }^{26}$, where an electron microscope images electrons that are simultaneously photoionized by attosecond pulses and accelerated in the local field of an infrared pulse. Coherent detection of high harmonics will enable a new generation of plasmon sensors of unprecedented sensitivity, in which small phase shifts of the resonance herald tiny variations in the environment surrounding the antennas.

Third, an inhomogeneous field will accelerate electron-hole pairs differently throughout the volume and even between successive laser half-cycles, since the electrons and holes follow oppositely directed paths. If the variation in the field is sufficient across the path length of the electron and the hole, the inversion symmetry is broken, allowing even harmonics ${ }^{30}$ to be produced. In fact, high harmonics can be thought of as a quantum sensor for weak fields or for field inhomogeneity. We do not observe emission of even harmonics. As discussed in the Supplementary Information, the opposite field gradients at the two ends of each antenna average the even harmonic signal in the far field to zero, and the asymmetry on either side of the antennas is predicted to be too small to yield appreciable even harmonic emission. The spatial average would be removed by imaging single nano-antennas with a sufficiently high NA lens, or by designing asymmetric antennas. In addition, the effect of the anisotropic field will be enhanced with a longer wavelength driver. We predict that at $3.7 \mu \mathrm{m}$ the even harmonic signal from the dominant short-trajectory electron-hole pairs will reach $\sim 3 \%$ of the nearest odd harmonic.

To conclude, we have observed non-perturbative harmonic generation from crystalline bulk $\mathrm{Si}$ assisted by plasmonic field enhancement from an array of monopole nano-antennas. Using solids overcomes the difficulty of generating macroscopic emission with low-density gas-a scheme that was tried in previous experiments $^{2-4}$. Harmonic emission is sensitive to the orientation of the incident linear laser polarization with respect to the major axis of the antennas. This effect can be exploited to engineer the polarization of the harmonic beam in the near field. For example, circularly polarized harmonics could be obtained by illuminating with a circularly polarized driver two overlapping arrays of antennas, with their major axis perpendicular to one another. Each antenna array will couple to the component of the electric field that aligns with the antenna's major axis, therefore with a quartercycle delay between the two arrays. This delay is inherited by the harmonics (multiplied by their order). Overlapping the emission from the two arrays will result in circularly polarized odd-order 
harmonics. Complicated geometries, such as metasurfaces ${ }^{31}$, will allow even more precise control of many properties of highharmonic beams, such as amplitude and orbital angular momentum.

Looking forward, light can be confined to volumes smaller than a few cubic nanometres ${ }^{32}$. In this regime, the atomic arrangement of the atoms of the antenna becomes relevant. Such tight confinement provides an opportunity to control strong-field excitation at the level of a single unit cell. This will be a valuable tool to address several conceptual issues in high-harmonic generation from solids, such as the role played by the relative diffusion of the electron and the hole, the plausibility of collisions between neighbouring electrons and holes, and the effect of boundaries and impurities on propagating electron-hole pairs. Combining solid-state technology with strongfield physics, high-harmonic generation can be engineered at will.

\section{Methods}

Methods, including statements of data availability and any associated accession codes and references, are available in the online version of this paper.

\section{Received 3 October 2016; accepted 8 March 2017; published online 3 April 2017}

\section{References}

1. Stockman, M. I. Nanoplasmonics: the physics behind the applications. Phys. Today 64, 39-44 (February, 2011).

2. Kim, S. et al. High-harmonic generation by resonant plasmon field enhancement. Nature 453, 757-760 (2008).

3. Park, I.-Y. et al. Plasmonic generation of ultrashort extreme-ultraviolet light pulses. Nat. Photon. 5, 677-681 (2011).

4. Park, I.-Y. et al. Generation of EUV radiation by plasmonic field enhancement using nano-structured bowties and funnel-waveguides. Ann. Phys. (Leipz.)525, 87-96 (2013).

5. Sivis, M. et al. Nanostructure-enhanced atomic line emission. Nature 485, E1-E3 (2012).

6. Sivis, M. et al. Extreme-ultraviolet light generation in plasmonic nanostructures. Nat. Phys. 9, 304-309 (2013).

7. Raschke, M. B. High-harmonic generation with plasmonics: feasible or unphysical? Ann. Phys. (Leipz.)525, A40-A42 (2013).

8. Feist, J., Homer Reid, M. T. \& Kling, M. F. Nanoplasmonic near-field synthesis. Phys. Rev. A 87, 033816 (2013).

9. Stewart, M. E. et al. Nanostructured plasmonic sensors. Chem. Rev. 108, 494-521 (2008).

10. Cingöz, A. et al. Direct frequency comb spectroscopy in the extreme ultraviolet. Nature 482, 68-71 (2012).

11. Li, X. F. et al. Multiple-harmonic generation in rare gases at high laser intensity. Phys. Rev. A 39, 5751-5761 (1989).

12. Pfeifer, T., Spielmann, C. \& Gerber, G. Femtosecond x-ray science. Rep. Prog. Phys. 69, 443-505 (2006).

13. Ghimire, S. et al. Observation of high-order harmonic generation in a bulk crystal. Nat. Phys. 7, 138-141 (2011).

14. Schubert, O. et al. Sub-cycle control of terahertz high-harmonic generation by dynamical Bloch oscillations. Nat. Photon. 8, 119-123 (2014).
15. Luu, T. T. et al. Extreme ultraviolet high-harmonic spectroscopy of solids. Nature 521, 498-502 (2015).

16. Vampa, G. et al. Generation of high harmonics from silicon. Preprint at http://arXiv.org/abs/1605.06345 (2016).

17. Han, S. et al. High-harmonic generation by field enhanced femtosecond pulses in metal-sapphire nanostructure. Nat. Commun 7, 13105 (2016).

18. Boyd, R. W. Nonlinear Optics (Academic, 2003).

19. Pfullmann, N. et al. Bow-tie nano-antenna assisted generation of extreme ultraviolet radiation. New J. Phys. 15, 093027 (2013).

20. Pfullmann, N. et al. Nano-antenna-assisted harmonic generation. Appl. Phys. B 113, 75-79 (2013).

21. Guler, U., Shalaev, V. M. \& Boltasseva, A. Nanoparticle plasmonics: going practical with transition metal nitrides. Mater. Today 18, 227-237 (April, 2015).

22. Barwick, B., Flannigan, D. J. \& Zewail, A. H. Photon-induced near-field electron microscopy. Nature 462, 902-906 (2009).

23. Kawata, S., Inouye, Y. \& Verma, P. Plasmonics for near-field nano-imaging and superlensing. Nat. Photon. 3, 388-394 (2009).

24. Zhang, B. et al. High contrast 3D imaging of surfaces near the wavelength limit using tabletop EUV ptychography. Ultramicroscopy 158, 98-104 (2015).

25. Chapman, H. N. et al. Femtosecond diffractive imaging with a soft-X-ray free-electron laser. Nat. Phys. 2, 839-843 (2006).

26. Förg, B. et al. Attosecond nanoscale near-field sampling. Nat. Commun. 7, 11717 (2016).

27. Kim, K. T. et al. Petahertz optical oscilloscope. Nat. Photon. 7, 958-962 (2013).

28. Dudovich, N. et al. Measuring and controlling the birth of attosecond XUV pulses. Nat. Phys. 2, 781-786 (2006).

29. Vampa, G. et al. Linking high harmonics from gases and solids. Nature 522, 462-464 (2015).

30. Ciappina, M. F. et al. High-order-harmonic generation from inhomogeneous fields. Phys. Rev. A 85, 033828 (2012).

31. Yu, N. \& Capasso, F. Flat optics with designer metasurfaces. Nat. Mater. 13, 139-150 (2014)

32. Barbry, M. et al. Atomistic near-field nanoplasmonics: reaching atomic-scale resolution in nanooptics. Nano Lett. 15, 3410-3419 (2015).

\section{Acknowledgements}

We thank D. Crane and B. Avery for technical support. G.V. thanks M. Sivis for insightful discussions. This material is based on work supported by the Air Force Office of Scientific Research under award number FA9550-16-1-0109 and the AFOSR MURI grant number FA9550-15-1-0037. The authors also acknowledge financial support from the NRC, NSERC and CFI/ORF.

\section{Author contributions}

G.V. and P.B.C. conceived the experiment; G.V and T.J.H. performed the high-harmonic measurements; B.G.G. and S.S.M. designed the nano-antennas; B.G.G., A.O. and E.L.-S. fabricated the antennas; A.S. and A.Y.N. maintained the laser source; D.M.V., P.B.C. and P.B. supervised the experiment; all authors contributed to the manuscript.

\section{Additional information}

Supplementary information is available in the online version of the paper. Reprints and permissions information is available online at www.nature.com/reprints. Publisher's note: Springer Nature remains neutral with regard to jurisdictional claims in published maps and institutional affiliations. Correspondence and requests for materials should be addressed to G.V. or P.B.C.

\section{Competing financial interests}

The authors declare no competing financial interests. 


\section{Methods}

Fabrication of the antennas. Several square monopole arrays were fabricated by electron-beam lithography, metal evaporation and lift-off on the (001) surface of a 500-nm-thick single-crystal silicon film (http://www.mtixtl.com/ sossilicononsapphire-2.aspx). The silicon was grown on an R-plane 500-nm-thick sapphire substrate. The Si/sapphire substrate was first annealed at a temperature of $200^{\circ} \mathrm{C}$ in ambient conditions for two hours. Immediately after cool down, two layers of polymethyl methacrylate (PMMA), each with a thickness of $40 \mathrm{~nm}$, were spun on the substrate to produce a re-entrant electron-beam resist bi-layer. PMMA $495 \mathrm{~A} 2$ and $950 \mathrm{~A} 2$ were used for the first and second layer, respectively. Both layers were baked at $180^{\circ} \mathrm{C}$ for one hour and cooled to room temperature before any further processing. The nano-antennas were patterned by electron-beam lithography at $30 \mathrm{keV}$ followed by a one-hour bake at $95^{\circ} \mathrm{C}$. The samples were developed in MIBK/IPA 1:3 at $20^{\circ} \mathrm{C}$. A 2- $\AA$-thick chromium adhesion layer was deposited directly on the substrate followed by evaporation of $200 \AA$ of gold, both using electron-beam evaporation. The metal lift-off took place in an acetone bath at $40^{\circ} \mathrm{C}$, which was sonicated at $30 \mathrm{kHz}$ for approximately one minute. Figure 1 shows a scanning electron micrograph of a nano-antenna array.

Generation and detection of high harmonics. An optical parametric amplifier (Light Conversion OPA TOPAS-Prime) is pumped with a titanium sapphire femtosecond regenerative amplifier (Coherent Legend Elite Cryo) and delivers infrared laser pulses of $100 \mathrm{fs}$ duration with a central wavelength of $2.1 \mu \mathrm{m}$ at
$10 \mathrm{kHz}$ repetition rate. The beam is spatially filtered with a diamond pinhole of $150 \mu \mathrm{m}$ diameter and refocused into the silicon sample with a magnification of $2 / 3$. The beam waist on the sample is comparable to the size of a nano-antenna array $(100 \mu \mathrm{m})$. The average power of the laser system is significantly attenuated from $P=150 \mathrm{~mW}$ to the required values of $<1 \mathrm{~mW}(<100 \mathrm{~nJ}$ per pulse) by a combination of a spatial filter and a small aperture placed before the filter. Control over the power is achieved by slightly varying the aperture size. The waist on the sample is unaffected since it is a fixed fraction of the pinhole size.

Harmonics with photon energies above the direct bandgap of $\mathrm{Si}$ (at $3.4 \mathrm{eV}$ ) are strongly absorbed. Because the region of field enhancement extends only a few nanometres below the Si surface, to detect these harmonics the Si film must face the detector. The projection of the sapphire $c$ axis on the surface aligns to the (100) direction of the Si crystal, and therefore shows birefringence for the desired output polarization parallel to the (110) direction. We used a combination of a half-wave and a quarter-wave plate to ensure that the polarization exiting the sapphire substrate and entering the Si film is linear and aligned along the (110) direction.

The harmonics are detected by focusing them through the slit of a visible-ultraviolet spectrometer from Ocean Optics (model USB2000+).

Data availability. The data that support the plots within this paper and other findings of this study are available from the corresponding author on reasonable request. 